\title{
The Parasite Extinction Assessment \& Red List: an open-source, online biodiversity database for neglected symbionts
}

${ }_{5}$ Colin J. Carlson*1 ${ }^{\dagger}$, Oliver C. Muellerklein ${ }^{1 \dagger}$, Anna J. Phillips ${ }^{2}$, Kevin R. Burgio ${ }^{3}$, Giovanni Castaldo ${ }^{1}$, Carrie A. Cizauskas ${ }^{1}$, Graeme S. Cumming ${ }^{4}$, Tad A. Dallas ${ }^{5}$, Jorge Doña ${ }^{6}$, Nyeema Harris ${ }^{7}$, Roger Jovani ${ }^{6}$, Zhongqi Miao ${ }^{1}$, Heather Proctor ${ }^{8}$, Hyun Seok Yoon ${ }^{1}$, Wayne M. Getz ${ }^{1,9}$

*For correspondence: cjcarlson@berkeley.edu (CJC); omuellerklein@berkeley.edu (OCM)

${ }^{\dagger}$ These authors contributed equally 1 to this work.
${ }^{1}$ Department of Environmental Science, Policy, and Management, University of California Berkeley; Berkeley, CA, United States; ${ }^{2}$ Department of Invertebrate Zoology, National Museum of Natural History, Smithsonian Institution; Washington, DC, United States; ${ }^{3}$ Department of Ecology and Evolutionary Biology, University of Connecticut; Storrs, CT, United States; ${ }^{4}$ ARC Centre of Excellence in Coral Reef Studies, James Cook University; Townsville, Queensland, Australia; ${ }^{5}$ Department of Environmental Science and Policy, University of California; Davis, CA, United States; ${ }^{6}$ Department of Evolutionary Ecology, Estación Biológica de Doñana (CSIC); Americo Vespucio s/n, E-41092 Sevilla, Spain; ${ }^{7}$ Ecology and Evolutionary Biology, University of Michigan; Ann Arbor, MI, United States; ${ }^{8}$ Department of Biological Sciences, University of Alberta; Edmonton, Alberta, Canada; ${ }^{9}$ School of Mathematical Sciences, University of KwaZulu-Natal; South Africa. Abstract Parasite conservation is a rapidly growing field at the intersection of ecology, epidemiology, parasitology, and public health. The overwhelming diversity of parasitic life on earth, and recent work showing that parasites and other symbionts face severe extinction risk, necessitates infrastructure for parasite conservation assessments. Here, we describe the release of the Parasite Extinction Assessment \& Red List (PEARL) version 1.0, an open-access database of conservation assessments and distributional data for almost 500 macroparasitic invertebrates. The current approach to vulnerability assessment is based on range shifts and loss from climate change, and will be expanded as additional data (e.g., host-parasite associations and coextinction risk) is consolidated in PEARL. The web architecture is also open-source, scalable, and extensible, making PEARL a template for more efficient red listing for other high-diversity, data-deficient groups. Future iterations will also include new functionality, including a user-friendly open data repository and automated assessment and re-listing. 


\section{Introduction}

Parasitism is one of the most common forms of life on Earth, if not, by species totals, the most common (Larsen et al., 2017; Dobson et al., 2008). Even excluding microparasites-such as some bacteria, viruses, and protozoans-the remaining diversity of macroparasites (e.g., helminths, leeches, lice, ticks, fleas, and mites) is staggering and comparatively understudied in ecology. Basic questions remain open at the intersection of parasitology with other fields like community ecology (Johnson et al., 2015), evolutionary ecology (Morand, 2015), macroecology (Stephens et al., 2016), and climate change biology (Brooks and Hoberg, 2007; Altizer et al., 2013; Cizauskas et al., 2017). As the field of disease ecology has matured, a growing body of work has shown that parasites are a critical part of ecosystems, acting as regulators of food webs and host populations, and serving an important role in energy flow through trophic levels (Dunne et al., 2013). The increasinglyapparent benefits of parasites make a case for their recognition as an important neglected target for conservation (Whiteman and Parker, 2005; Pizzi, 2009; Carlson et al., 2013; Gómez and Nichols, 2013; Dougherty et al., 2016; Rocha et al., 2016), especially given that parasitic life cycles are already known to be particularly extinction-prone due to cascading co-extinctions with hosts (Durden and Keirans, 1996; Dunn et al., 2009; Dallas and Cornelius, 2015; Farrell et al., 2015). With recent work showing that climate change and coextinction combined could threaten one in every three helminth parasite species (Carlson et al., 2017), frameworks to assess and catalog parasite extinction risk are urgently needed.

While institutions such as the IUCN have spent decades developing centralized frameworks for prioritizing the conservation of free-living biodiversity, parasites are rarely included in mainstream assessments; for example, only two animal macroparasites are listed on the IUCN Red List (Hematopinus oliveri, the pygmy hog louse, and Hirudo medicinalis, the medicinal leech). The under-representation of parasites speaks to broader deficiencies in IUCN invertebrate assessments, but also to the comparative bias against parasites in conservation, which has conventionally treated parasites and disease as synonymous (Dougherty et al., 2016). Mainstreaming parasites into conservation requires researchers to address a number of additional factors: the independent extinction risk of parasites as well as coextinction risk, the degree of host specificity, the modes and efficiency of transmission, the possibility for unintended consequences to wildlife or human health, the cost-effectiveness of parasite conservation as a compatible goal with host conservation, and the feasibility of ex situ conservation (Dougherty et al., 2016).

At the most basic level, incorporating parasite conservation measures into existing free-living species' conservation plans can prevent accidental or deliberate loss of affiliates (Jørgensen, 2015), e.g. the extinction of the California condor's louse Colpocephalum californianus, or the black-footed ferret louse Neotrichodectes sp. (Stringer and Linklater, 2014). Some key assessments have been made for parasites of high-profile hosts like the black-footed ferret (Gompper and Williams, 1998) or the Tasmanian devil (Wait et al., 2017), but more expansive assessments are rare. Recent work has pushed to embrace a broader perspective on symbiosis within parasitology (Jovani et al., 2017), and in the context of global change biology, we believe this is an important step towards effective conservation. Symbionts as a broad group face a common set of challenges, and the same conservation measures that make sense for parasites are part of a broader shift towards symbiont-conscious conservation.

Rising interest in parasite conservation comes at a time when open data in parasitology is rapidly expanding; while host-parasite association data have historically been available from scientific collections or online databases (Strona and Lafferty, 2012; Dallas, 2016), only in the last few years have major sources such as the U.S. National Parasite Collection (Lichtenfels et al., 1992; Carlson et al., 2017) or Global Mammal Parasite Database (Stephens et al., 2017) been updated to include the detailed spatial data that are critical for conservation assessments. Expanding existing repositories, and improving access to collections data, is already a key part of ongoing work bridging the gap between parasitology and other fields like epidemiology, disease ecology, and conservation 
(DiEuliis et al., 2016). For parasite conservation to be operational and actionable in the shortest term, researchers currently need a detailed and dedicated bioinformatic repository for the explicit purpose of centralizing data on population trends, extinction risk, distributions, and conservation efforts. Here, we present the Parasite Extinction Assessment \& Red List (PEARL: pearl.berkeley.edu), the first standalone global parasite conservation assessment, database, and web interface.

\section{PEARL version 1.0}

The Parasite Extinction Assessment \& Red List compiles the preliminary work of the Parasite Extinction Research Project (2013-2016). The core study (Carlson et al., 2017) accomplished three tasks:

1. The georeferencing of the U.S. National Parasite Collection (USNPC), and the compilation of the most detailed occurrence dataset for macroparasites currently available.

2. The estimation of global parasite extinction risk: an estimated $5-10 \%$ of species face direct extinction risk from climate change, while up to $30 \%$ of helminths face a combined threat from coextinction and climate change.

3. The preliminary release of PEARL version 0.1 , a prototype with a static interface, PDF maps, and no search functionality.

Building on PEARL v0.1, the full PEARL v1.0 was released on August 11, 2017 and features an opensource web architecture, which allows a more flexible interface and tool to access the underlying open-access database. Here we formalize the documentation for PEARL v1.0, explaining the updated mechanics of PEARL as a new, standalone form of red listing for parasites and other symbionts; and to outline the use of the framework in the inclusion of parasites in conservation research, and more generally for invertebrate conservation.

\section{The Assessment}

As a scientific resource, PEARL serves two main purposes: an extinction assessment, which compiles the extinction risk of enough species that global parasite extinction rates from climate change can be measured; and a red list, analogous to the IUCN Red List but focused specifically on measuring the vulnerability of macroparasitic species. The species currently included in the assessment fall into eight major groups: helminth endoparasites (acanthocephalans, cestodes, nematodes, and trematodes) and arthropod ectoparasites (fleas, lice, mites, and ticks). The term parasites is used broadly both here and in the underlying premise of PEARL. While the focus of the assessment is parasitic species, in the overarching goal of mainstreaming parasites into conservation (Dougherty et al., 2016), several species and groups are included that are not strictly parasitic. For example, vane-dwelling feather mites (Acariformes: Analgoidea, Pterolichoidea), like many other symbionts, may contextually change roles between parasitism and commensalism or mutualism (Galván et al., 2012); within other significant groups in our study, parasitism has secondarily evolved multiple times (Dorris et al., 1999; Bochkov and Mironov, 2013).

The vulnerability of species is measured based on projected range loss in the face of climate change, which was forecasted through the use of ecological niche modeling (Corlson et al., 2017). Eighteen climate change scenarios are included in that assessment for 457 non-marine macroparasitic species, and extinction risk is estimated based on those projected rates of habitat loss. These are translated into "red listing categories" based on estimated percent range loss in the next 50 years (i.e., by 2070):

- Critically endangered (CR): projected decline by $\geq 80 \%$ in 50 years

- Endangered (EN): projected $50-79 \%$ decline in 50 years

- Vulnerable (VU): projected $25-49 \%$ decline in 50 years

- Least Concern (LC): $<25 \%$ decline in 50 years 
The assessment is designed to be fully transparent about accuracy and bias; accuracy metrics for niche modeling (the true skill statistic and the area under the receiver-operator curve), as well as categorical measures of data quality, are presented alongside distribution models. We developed two data quality metrics based on percentiles within our dataset. Data coverage is based on sample size:

\begin{tabular}{ccc}
\hline Coverage & Percentile & Occurrence Points \\
\hline "Poor" & $0-25$ & $0-28$ \\
"Fair" & $25-50$ & $29-42$ \\
"Good" & $50-75$ & $43-80$ \\
"Excellent" & $75-100$ & $81-3289$
\end{tabular}

Data uncertainty is based on the mean uncertainty radius of every point from manual georeferencing; some species only had GPS-identified data, which were classified as zero uncertainty from georeferencing:

\begin{tabular}{ccc}
\hline Uncertainty & Percent & Distance $(\mathrm{km})$ \\
\hline "Perfect" & - & 0 \\
"Excellent" & $0-25$ & $0-5.1$ \\
"Good" & $25-50$ & $5.1-6.5$ \\
"Fair" & $50-75$ & $6.5-7.9$ \\
"Weak" & $75-100$ & $7.9-21.3$ \\
\hline
\end{tabular}

By presenting quality as a mainstreamed part of the assessment, we improve transparency, better inform practitioners relying on these data for any applied research, and clearly identify species for which improved data collection is needed (i.e., more precise locality data).

\section{The Web Interface}

PEARL is an open-source web app that builds on several frontend and backend APIs and novel software libraries in both the Python and Javascript programming languages. In the backend, PEARL builds upon a Python 3.5 based web framework developed by Z. Miao and O. Muellerklein called Extensible Web App Interactive Mapping, EWAIM on Github ${ }^{1}$. EWAIM incorporates continuous unit testing, a basic yet extensible library for server-side (i.e. backend) data analysis, and native integration with spatial data structures through PostGIS / PostgreSQL or SQLite. PEARL's use of the underlying EWAIM server-side web framework allows near endless GIS functionality to be used on spatial or time series data.

In the frontend, PEARL uses standard GET / POST events to process user events to and from the server-side application, allowing interactivity with the backend database via species maps and other functionality in the various web pages / interfaces. Building upon EWAIM, PEARL handles user events to and from web pages through Flask protocol, a Python based extensible web microframework. Within the frontend components of PEARL a range of mapping APIs are called. Frontend libraries and API used in PEARL include:

- D3.js - Data Driven Documents: ${ }^{2}$ an open-source JavaScript library for dynamic, interactive data visualizations

- Leaflet API: ${ }^{3}$ an open-source JavaScript library that allows interactive mapping of PEARL tilemaps and provides a mobile-friendly design

\footnotetext{
${ }^{1}$ EWAIM on Github: https://github.com/Thru-Echoes/ewaim-webapp

2D3.js homepage: https://d3js.org/

${ }^{3}$ Leaflet API homepage: http://leafletjs.com/
} 


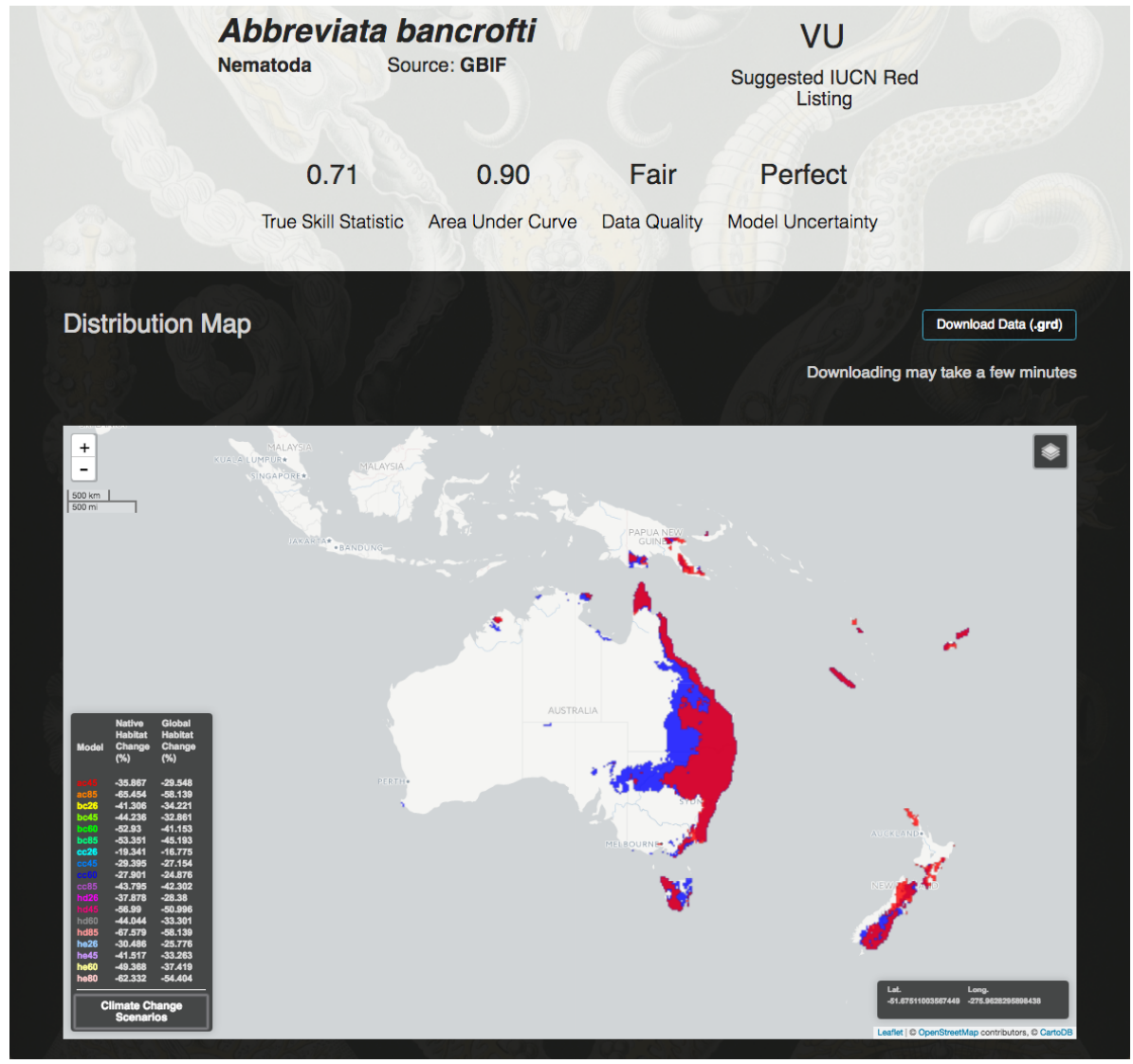

Figure 1. The online interface to PEARL v1.0, illustrated for Abbreviata bancrofti (Physalopteridae; Irwin-Smith, 1922), a nematode parasite of the Australian leaf-tailed gecko, Phyllurus platurus (Reptilia: Gekkonidae). Results include the current distribution of the nematode (blue) and 18 future climate scenarios. Above, information about the entry is available, including data sources, and model accuracy metrics.

- CARTO Maps API: ${ }^{4}$ an open-source engine that is scalable and extensible, powers a range of basemaps, and interacts with Leaflet, Google Earth Engine, and CARTO SQL data structures

PEARL, as a web app and open-source software, is fully documented and available publicly as a Github repository ${ }^{5}$ with version releases and community-based issues tracked accordingly. The underlying raster files can be downloaded in grid file (.grd) format directly from species pages, and R- and Python-based APIs are currently in development that will allow users to pull data from multiple species at once. Future iterations of PEARL development include, but are not limited to, a more robust mobile-friendly structure; public data uploads and downloads through user login and associated profiles; and the incorporation of a novel dynamic, real-time algorithm that would automate generating and rendering distribution models and associated data analysis based on user data contributions (see Dynamic Updating section below).

\section{Extending the Framework}

The purpose of PEARL is to create a stable platform for parasite conservation that allows continuous improvements by existing teams, and that can incorporate future collaborations from other researchers both in PEARL development and database-building. The full expanded platform makes the interface, and underlying database, extensible in a number of important ways that will be useful both for the future of PEARL, and as a template for the broader problem of invertebrate

${ }^{4}$ CARTO homepage: https://carto.com/

${ }^{5}$ PEARL Github repository: https://github.com/Thru-Echoes/PEARL1.0 
conservation assessments. After the release of version 1.0, a number of scheduled advances are planned for PEARL over the coming years. We detail five here:

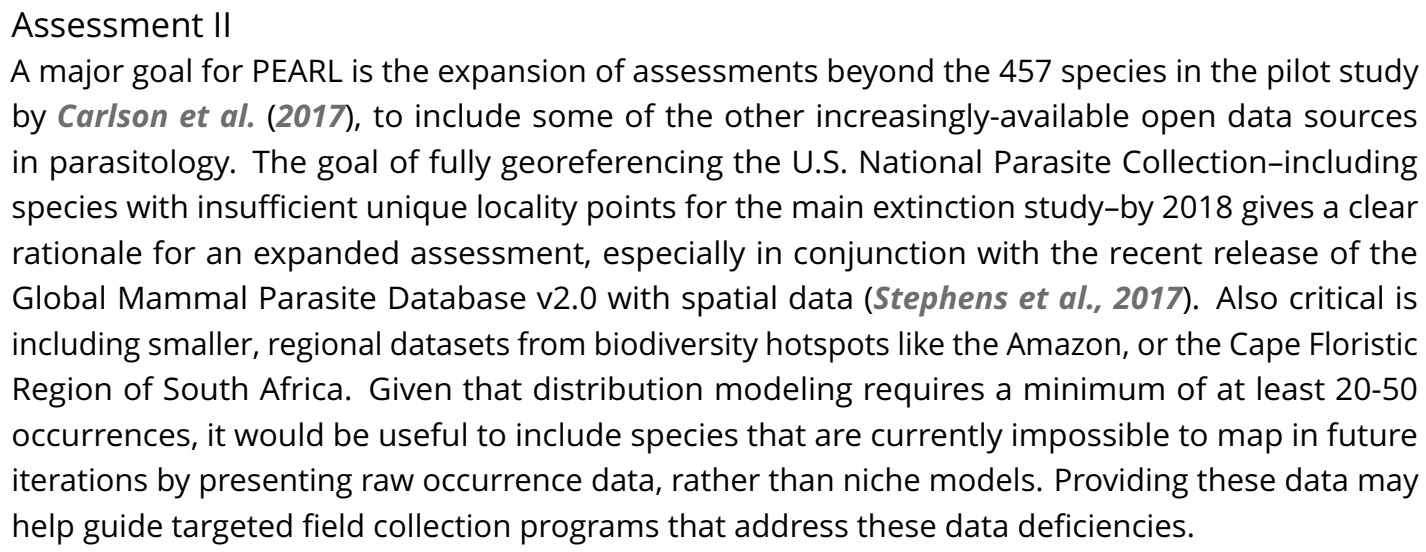

\section{Reconsidered Criteria}

Red listing parasites-or, in fact, any dependent species-poses a more severe methodological problem than already-challenging work on free-living species. In particular, well-designed criteria must accommodate the tremendous diversity of symbiotic groups, and rescale important metrics of viability to an appropriate level; but effective criteria must also (presumably) include information about the vulnerability of hosts. The criteria underlying assessment version 1.0 only indirectly addressed the first of these two challenges, by presenting a radically simplified version of the Thomas et al. (2004) criteria, which were already reduced from the IUCN Red List criteria.

For comparison, the criteria used in Thomas et al. (2004) are designed to correspond directly to projected extinction risk:

- Extinct (EX): species with a projected future area of zero (100\% of species assumed to be committed to eventual extinction)

- Critically endangered (CR): projected future distribution area $<10 \mathrm{~km}^{2}$, or decline by $\geq 80 \%$ in 50 years (species assigned a $75 \%$ chance of extinction)

- Endangered (EN): projected area 10-500 km², or 50-79\% decline in 50 years (species assigned a $35 \%$ chance of extinction)

- Vulnerable (VU): projected area 501-2,000 km², or > 50\% decline in 100 years on the basis of linear extrapolation of 50-year projection (species assigned a 15\% chance of extinction)

- Not Threatened (NT): $0 \%$ extinction risk, no area loss

In the Carlson et al. (2017) study and PEARL v0.1 (and v1.0), these criteria are reduced to percentagebased criteria only:

- Critically endangered (CR): projected decline by $\geq 80 \%$ in 50 years

- Endangered (EN): projected $50-79 \%$ decline in 50 years

- Vulnerable (VU): projected $25-49 \%$ decline in 50 years

- Least Concern (LC): $<25 \%$ decline in 50 years

The inadequacy of these criteria is a key point articulated by Carlson et al. (2017), and we return to it here to note that it offers only the coarsest level of possible resolution for categorizing extinction risk. More detailed criteria are needed that incorporate risk factors like small ranges (with minimal projected declines), but parasite conservation has yet to develop meaningful benchmarks for these criteria; what is a "small range" for a parasitic species? Is a small range for a trophically-transmitted nematode the same size as a small range for a tick with a single common host? The role of microclimate and heterogeneity within ranges, or of habitat selection and dispersal patterns of hosts, further complicates this problem. Similarly, Dougherty et al. (2016) highlighted the need for 
advances in population viability analysis for parasites, such that concepts like "minimum viable population" can be readily applied, and included in these criteria.

\section{Better Host \& Parasite Bioinformatics}

Host and symbiont extinction risk are fundamentally linked, and conservation of parasites and other symbionts cannot exist in the absence of detailed host information. For ticks and feather mites, underlying data from the Carlson et al. (2017) study contains host association data that can be mainstreamed into future versions. Moreover, access to portals like the helminthR package in $\mathrm{R}$ (Dallas, 2016) will make it possible to compile detailed information on host-parasite associations for helminths, but these data lack information about the life stage at which different hosts are relevant. Adding life stage-structured data to host-parasite associations will be a key part of PEARL expansion, especially given that host-range disjunctions might be a substantial pressure on parasites in a changing climate (Pickles et al., 2013; Cizauskas et al., 2017). A new database published this year makes significant strides towards aggregating life cycle data for acanthocephalans, nematodes, and cestodes (Benesh et al., 2017); compiling that data for every species in our study will still likely require the concerted effort of researchers contributing to the expansion of the PEARL database.

The integration of host-parasite association data is an especially sensitive matter in the design of actual parasite conservation schemes. The potential for conflict between parasite conservation and the broader goals of wildlife and human health has already been noted by Dougherty et al. (2016), and a fundamental tenet of effective parasite conservation is attention to potential unanticipated consequences for conservation or public health. Parasites with zoonotic potential or that act as vectors of zoonoses are an especially difficult case, as they may be the target of eradication campaigns simultaneous to conservation efforts for closely related parasites. Developing an aggregated bioinformatic infrastructure that serves both purposes will support parasite research in diverse realms, and facilitate the work of public health practicioners and conservation managers alike. Consequently, future assessments should not only include wildlife (and domestic) host associations, but also detailed information on the known zoonotic potential of every species.

\section{Integrating Genomic Data}

The increasing availability of genomic data associated with the continuous improvements in sequencing and bioinformatics (Stephens et al., 2015), and with global initiatives such as the Earth BioGenome project (EBP; Pennisi (2017)), is becoming a huge source of data for conservation assessments (Pauls et al., 2013; Ikeda et al., 2017; Razgour et al., 2017). Genomic data can be programmatically gathered from massive databases, such as the NCBI Genome database ${ }^{6}$ and the European Nucleotide Archive (ENA ${ }^{7}$ ). Notwithstanding, given the bias against symbiont genomes (Del Campo et al., 2014), PEARL may need dedicated projects to generate genomic data for the species already included. Doing so open the doors to a number of important new analysies, such as genetically informed ecological niche models (ENMs; Marcer et al. (2016); Ikeda et al. (2017)) following integrative frameworks (Razgour et al., 2017). In this way, assessments can include measures based on neutral and adaptive genomic information to assess the sensitivity of species to environmental variables associated with global change. For these computationally challenging purposes, PEARL will likely require of an increase in computational resources (Hayden, 2015).

\section{Dynamic Updating}

One strength of PEARL's dynamic interface is the potential for continuously-updating red listing, which updates existing species assessments and adds new ones in real-time as new data are contributed by researchers around the world. In an upcoming release, we hope to include an automated tool for continuous integration of new data and assessments, in which submitted spatial data automatically augments the existing global database. This workflow will pave the way for future

\footnotetext{
${ }^{6}$ http://www.ncbi.nlm.nih.gov/genome/

${ }^{7}$ http://www.ebi.ac.uk/ena
} 
conservation approaches by allowing dynamically updated red listing, via continuous integration of data into self-updating niche models and respective quality metrics. PEARL can serve as a launching point for an alternative red listing protocol that incorporates machine learning methods to evaluate conservation status at the community or global level (e.g. see recent work by Darrah et al. (2017)), rather than on a manual species-to-species basis (like most current efforts operate), something that will likely be helpful in red listing the 300,000 estimated species of helminths alone.

\section{Application to Other Conservation Efforts}

PEARL is designed to be a template for more successful rapid assessment of conservation risk and status for invertebrates and other difficult-to-profile groups, especially other types of symbionts and coextinction-prone affiliate species. All code for PEARL is publicly available, allowing the rapid and easy development of parallel tools for non-parasitic groups, and encouraging a broader culture of open, reproducible science in conservation. The Github repository contains a detailed user-guide to installing local and server-side instances of PEARL and its underlying web architecture across a number of operating systems, making it easily adaptable for a diverse range of ecological projects. Developing better, broader frameworks for invertebrate conservation could be substantially accelerated with readily available, open-source frameworks for red listing that allow more decentralized data collection and assessment. Data deficiencies for invertebrates are overwhelming (Clausnitzer et al., 2009; Régnier et al., 2015), and despite the priority put on red listing insects, there is concern that hyper-diverse groups like the insects will never be described thoroughly enough that conservation assessments can keep pace with extinction rates (Warren et al., 2007). Decentralizing the red listing process, and enabling smaller assessments as data are collected, is an important step to protecting not just parasites, but all symbionts and other neglected or understudied groups.

\section{Acknowledgments}

We would like to thank the generous contributions of time and effort to the Parasite Extinction Research Project by our broader team, including Christopher Clements, Eric Dougherty, Sarah Fourby, Nicole Kula, and Sergey Mironov. We especially also thank Gary Casterline for invaluable server and file system assistance for PEARL.

\section{References}

Altizer S, Ostfeld RS, Johnson PT, Kutz S, Harvell CD. Climate change and infectious diseases: from evidence to a predictive framework. Science. 2013; 341(6145):514-519.

Benesh DP, Lafferty KD, Kuris A. A life cycle database for parasitic acanthocephalans, cestodes, and nematodes. Ecology. 2017; 98(3):882-882.

Bochkov A, Mironov S. Is parasitism of metazoa "a one-way ticket"? Entomological review. 2013; 93(9):11961206.

Bostock M, Ogievetsky V, Heer J. D3 Data-Driven Documents. IEEE Transactions on Visualization and Computer Graphics. 2011 Dec; 17(12):2301-2309. https://d3js.org/, doi: 10.1109/TVCG.2011.185.

Brooks DR, Hoberg EP. How will global climate change affect parasite-host assemblages? Trends in parasitology. 2007; 23(12):571-574.

Carlson CJ, Burgio KR, Dougherty ER, Phillips AJ, Bueno VM, Clements CF, Castaldo G, Dallas TA, Cizauskas CA, Cumming GS, Doña J, Harris NC, Jovani R, Mironov S, Muellerklein OC, Proctor HC, Getz WM. Parasite biodiversity faces extinction and redistribution in a changing climate. Science Advances. 2017; 3:e1602422.

Carlson CJ, Cizauskas CA, Burgio KR, Clements CF, Harris NC. The more parasites, the better? Science. 2013; 342(6162):1041-1041.

Cizauskas CA, Carlson CJ, Burgio KR, Clements CF, Dougherty ER, Harris NC, Phillips AJ. Parasite vulnerability to climate change: an evidence-based functional trait approach. Royal Society Open Science. 2017; 4(1):160535. 
Clausnitzer V, Kalkman VJ, Ram M, Collen B, Baillie JE, Bedjanič M, Darwall WR, Dijkstra KDB, Dow R, Hawking J, et al. Odonata enter the biodiversity crisis debate: the first global assessment of an insect group. Biological Conservation. 2009; 142(8):1864-1869.

Colwell RK, Dunn RR, Harris NC. Coextinction and persistence of dependent species in a changing world. Annual Review of Ecology, Evolution, and Systematics. 2012; 43:183-203.

Dallas T. helminthR: an R interface to the London Natural History Museum's host-parasite database. Ecography. 2016; 39(4):391-393.

Dallas T, Cornelius E. Co-extinction in a host-parasite network: identifying key hosts for network stability. Scientific reports. 2015; 5:13185.

Darrah SE, Bland LM, Bachman SP, Clubbe CP, Trias-Blasi A. Using coarse-scale species distribution data to predict extinction risk in plants. Diversity and Distributions. 2017; 23(4):435-447.

Del Campo J, Sieracki ME, Molestina R, Keeling P, Massana R, Ruiz-Trillo I. The others: Our biased perspective of eukaryotic genomes. Trends in Ecology and Evolution. 2014; 29(5):252-259. doi: 10.1016/j.tree.2014.03.006.

DiEuliis D, Johnson KR, Morse SS, Schindel DE. Opinion: Specimen collections should have a much bigger role in infectious disease research and response. Proceedings of the National Academy of Sciences. 2016; 113(1):4-7.

Dobson A, Lafferty KD, Kuris AM, Hechinger RF, Jetz W. Homage to Linnaeus: How many parasites? How many hosts? Proceedings of the National Academy of Sciences. 2008; 105(Supplement 1):11482-11489.

Dorris M, De Ley P, Blaxter M. Molecular analysis of nematode diversity and the evolution of parasitism. Parasitology today. 1999; 15(5):188-193.

Dougherty E, Carlson C, Bueno V, Burgio K, Cizauskas C, Clements C, Seidel D, Harris N. Paradigms for parasite conservation. Conservation Biology. 2016; 30(4):724.

Dunn RR, Harris NC, Colwell RK, Koh LP, Sodhi NS. The sixth mass coextinction: are most endangered species parasites and mutualists? Proceedings of the Royal Society of London B: Biological Sciences. 2009; 276(1670):3037-3045.

Dunne JA, Lafferty KD, Dobson AP, Hechinger RF, Kuris AM, Martinez ND, McLaughlin JP, Mouritsen KN, Poulin R, Reise $\mathrm{K}$, et al. Parasites affect food web structure primarily through increased diversity and complexity. PLoS biology. 2013; 11(6):e1001579.

Durden LA, Keirans JE. Host-parasite coextinction and the plight of tick conservation. American Entomologist. 1996; 42(2):87-91.

Farrell MJ, Stephens PR, Berrang-Ford L, Gittleman JL, Davies TJ. The path to host extinction can lead to loss of generalist parasites. Journal of Animal Ecology. 2015; 84(4):978-984.

Galván I, Aguilera E, Atiénzar F, Barba E, Blanco G, Cantó JL, Cortés V, Frías Ó, Kovács I, Meléndez L, et al. Feather mites (Acari: Astigmata) and body condition of their avian hosts: a large correlative study. Journal of Avian Biology. 2012; 43(3):273-279.

Gómez A, Nichols E. Neglected wild life: parasitic biodiversity as a conservation target. International Journal for Parasitology: Parasites and Wildlife. 2013; 2:222-227.

Gompper ME, Williams ES, Parasite conservation and the black-footed ferret recovery program. JSTOR; 1998.

Hayden EC. Genome researchers raise alarm over big data. Nature. 2015; doi: 10.1038/nature.2015.17912.

Ikeda DH, Max TL, Allan GJ, Lau MK, Shuster SM, Whitham TG. Genetically informed ecological niche models improve climate change predictions. Global Change Biology. 2017; 23(1):164-176. doi: 10.1111/gcb.13470.

Johnson PT, De Roode JC, Fenton A. Why infectious disease research needs community ecology. Science. 2015; 349(6252):1259504.

Jørgensen D. Conservation implications of parasite co-reintroduction. Conservation Biology. 2015; 29(2):602604.

Jovani R, Doña J, del Mar Labrador M, Serrano D. Opening the Doors of Parasitology Journals to Other Symbionts. Trends in Parasitology. 2017; . 
Larsen BB, Miller EC, Rhodes MK, Wiens JJ. Inordinate Fondness Multiplied and Redistributed: the Number of Species on Earth and the New Pie of Life. The Quarterly Review of Biology. 2017; 92(3):229-265.

Lichtenfels JR, Pilitt P, Hoberg EP. The US National Parasite Collection: a century of service. Journal of Parasitology. 1992; p. 924-929.

Marcer A, Méndez-Vigo B, Alonso-Blanco C, Picó FX. Tackling intraspecific genetic structure in distribution models better reflects species geographical range. Ecology and Evolution. 2016; 6(7):2084-2097. doi: 10.1002/ece3.2010.

Morand S. (macro-) Evolutionary ecology of parasite diversity: From determinants of parasite species richness to host diversification. International Journal for Parasitology: Parasites and Wildlife. 2015; 4(1):80-87.

Pauls SU, Nowak C, Bálint M, Pfenninger M. The impact of global climate change on genetic diversity within populations and species. Molecular Ecology. 2013; 22(4):925-946. doi: 10.1111/mec.12152.

Pennisi E. Sequencing all life captivates biologists. Science. 2017; 355(6328):894-895. doi: 10.1126/science.355.6328.894.

Pickles RS, Thornton D, Feldman R, Marques A, Murray DL. Predicting shifts in parasite distribution with climate change: a multitrophic level approach. Global change biology. 2013; 19(9):2645-2654.

Pizzi R. Veterinarians and taxonomic chauvinism: the dilemma of parasite conservation. Journal of Exotic Pet Medicine. 2009; 18(4):279-282.

Razgour O, Taggart JB, Manel S, Juste J, Ibáñez C, Rebelo H, Alberdi A, Jones G, Park K. An integrated framework to identify wildlife populations under threat from climate change. Molecular Ecology Resources. 2017; p. 1-14. doi: 10.1111/1755-0998.12694.

Régnier C, Achaz G, Lambert A, Cowie RH, Bouchet P, Fontaine B. Mass extinction in poorly known taxa. Proceedings of the National Academy of Sciences. 2015; 112(25):7761-7766.

Rocha CFD, Bergallo HG, Bittencourt EB. More than just invisible inhabitants: parasites are important but neglected components of the biodiversity. Zoologia (Curitiba). 2016; 33(3).

Stephens PR, Altizer S, Smith KF, Alonso Aguirre A, Brown JH, Budischak SA, Byers JE, Dallas TA, Jonathan Davies T, Drake JM, et al. The macroecology of infectious diseases: a new perspective on global-scale drivers of pathogen distributions and impacts. Ecology letters. 2016; 19(9):1159-1171.

Stephens PR, Pappalardo P, Huang S, Byers JE, Farrell MJ, Gehman A, Ghai RR, Haas SE, Han B, Park AW, et al. Global Mammal Parasite Database version 2.0. Ecology. 2017; 98(5):1476-1476.

Stephens ZD, Lee SY, Faghri F, Campbell RH, Zhai C, Efron MJ, lyer R, Schatz MC, Sinha S, Robinson GE. Big data: Astronomical or genomical? PLoS Biology. 2015; 13(7):1-11. doi: 10.1371/journal.pbio.1002195.

Stringer AP, Linklater W. Everything in moderation: principles of parasite control for wildlife conservation. BioScience. 2014; 64(10):932-937.

Strona G, Lafferty KD. FishPEST: an innovative software suite for fish parasitologists. Trends in parasitology. 2012; 28(4):123.

Thomas CD, Cameron A, Green RE, Bakkenes M, Beaumont LJ, Collingham YC, Erasmus BF, De Siqueira MF, Grainger A, Hannah L, et al. Extinction risk from climate change. Nature. 2004; 427(6970):145-148.

Wait LF, Peck S, Fox S, Power ML. A review of parasites in the Tasmanian devil (Sarcophilus harrisii). Biodiversity and Conservation. 2017; p. 1-18.

Warren MS, Bourn N, Brereton T, Fox R, Middlebrook I, Parsons MS. What have red lists done for us? The values and limitations of protected species listing for invertebrates. Insect conservation biology. 2007; p. 76-91.

Whiteman NK, Parker PG. Using parasites to infer host population history: a new rationale for parasite conservation. In: Animal Conservation forum, vol. 8 Cambridge University Press; 2005. p. 175-181. 